\title{
A New Medium for Matter and Light Waves
}

\author{
Ahmed Isam ${ }^{1}$ \\ Independent Researcher, Khartoum, Sudan
}

\begin{abstract}
A new medium for matter and light waves is proposed in this paper. This medium represents a modern version of the classical light's medium, the aether. The new version is called Sama aether, and it differs from the classical model in that both matter and light waves are propagating disturbances in this medium. This reveals a new and elegant kind of unification between the classical and the modern physical concepts. The new model is based on two simple postulates. The first is concerned with the contradiction in the experimental efforts, which aimed to detect any motion with respect to ether, and solving this contradiction simply by assuming that the ether is a multi-rest frames medium. The second postulate of the theory is concerned with resolving the contradictory wave-particle duality of matter and light by demonstrating that they are both propagating disturbances in the same physical entity. This is done by reviewing the multiplicity of energy forms in nature and showing that they are limited than was assumed. A major consequence of this is that gravity is revealed as a new form of energy, which is the third form of kinetic energy. Finally, two indirect evidences regarding the existence of ether are introduced. The first is concerned with the behavior of light regarding the findings of Thomas Young, and Michelson-Morley experiments. The second is concerned with the ability of ether to curve space-time intervals with motion, and the linkage between this effect and the source of inertial mass.
\end{abstract}

Keywords : Sama Aether; Speed of Light; Michelson-Morley’s Experiment; Multi-Rest Frames ;Wave-Particle Duality; Gravity ; The third form of kinetic energy; Rest Motion; Origin of Mass

\section{INTRODUCTION}

When a water wave is observed, what is seen is the rearrangement of the water's surface. Without the water, there would be no wave. A wave traveling on a string would not exist without the string. Sound waves could not travel from one point to another if there were no air molecules between the two points. A simple definition of a wave arises from the previous examples, is that a wave represents a propagating disturbance that moves from one point to another. The disturbance by itself can be viewed as a difference between initial and final states which are taken by a preexisting physical entity. This physical entity is called a medium. This concept is not restricted to the mechanical waves. In the early $20^{\text {th }}$ century, a new type of waves was assumed to be existed, which is the gravitational waves [1]. In their concept, they represent a propagating disturbance in the space-time itself. Therefore, in this case the space-time represents the medium for these waves, which is consistent with the simple definition of the wave.

Due to the wave properties of light, many physicists of the late 1800 s were convinced that light waves moved through a medium called the aether or ether. Today in physics the word of "ether" refers to many physical concepts, but the only concept which will be meant in this paper is that it represents a medium for light propagation.

Historically, the concept of ether had faced four ${ }^{1}$. The first was introduced by C. Huygens, when he hypothesized that light was a wave propagating through a medium called the ether. He described the ether as a medium that occupies all the universe, since it carries the light, therefore it should be existed everywhere, but his vision of ether and light was entirely mechanical[2].

The second shift was introduced by J.C. Maxwell when he showed that light can be considered as electromagnetic wave. He introduced the concept of the field to describe the nature of light. From this vision, the apparent need for a propagation medium for light waves can be seen by the fact that they consist of perpendicular electric (E) and magnetic (B) waves. The (E) waves consist of undulating dipolar electric fields, and all such dipoles appeared to require separated and opposite electric charges. Electric charge is an inextricable property of matter, so it appeared that some form of matter was required to provide the alternating current that would seem to have to exist at any point along the propagation path of the wave. Propagation of waves in a true vacuum would imply the existence of electric fields without associated electric charge, or of electric charge without associated matter. Several mechanical models of ether were proposed, and these models had to agree with the fact that the electromagnetic waves are

\footnotetext{
${ }^{1}$ Corresponding Author: ahmedisam777@gmail.com
} 
transverse waves. At this point the mechanical models of the ether had become magical: it had to be a fluid in order to fill space, but one that was millions of times more rigid than steel in order to support the high frequencies of light waves. It also had to be massless and without viscosity, otherwise it would visibly affect the orbits of planets. Additionally it appeared it had to be completely transparent, non-dispersive, incompressible, and continuous at a very small scale. From here it will become prominent that the traditional mechanical models of ether cannot survive, and as a result of this the ether has to shift from a mechanical into a non-mechanical physical entity. [3]

By proposing a non-mechanical structure of ether, which means that it is not composed of particles ( matter) and therefore energy that can be directly detected in the laboratory. This is by itself demonstrates the great difficulty for making any prediction from any proposed ether model that can lead us to a direct detection of the ether. But on the other hand it explains why the ether does not appear or interfere with the sensitive experiments of modern physics especially those of the EM phenomena as if they were performed in a true vacuum if the ether is really existed. This is in contrast with the disturbances that propagate through the ether which represents ether's waves that carry energy and therefore matter that are easily detected experimentally Therefore, for now, it seems that the possibility for detecting the ether will depend on the indirect methods which means the detection of the physical consequences regarding the existence of the medium based on the physical properties which are concluded from any proposed model of ether.

Defining the major historical steps in the development of any scientific concept may sometimes be a site of controversy between scientists. Therefore, this text represents only the author's prospect.

The third shift was performed in 1887 by Albert Michelson and Edward Morley when they performed their famous experiment at the Case School of Applied Science in Cleveland, Ohio[4]. Their experiment aimed to detect any motion of the earth with respect to ether. At that time their experiment was very sensitive to detect such an effect, but it failed. Its failure enhanced the Dutch physicist H. A. Lorentz to introduce what is today known as Lorentz's transformation[5][6]. His transformation allowed moving lengths to shrink in the same direction of motion. The transformation was successful in justifying the results of Michelson-Morley's Experiment. Also, this transformation was used by him and G. Fitzgerald in a new ether model which is known today as Lorentz's ether theory (LET)[3][7].

The final shift happened in 1905 when another theory was introduced by a German-American physicist named Albert Einstein. His new theory shared the same mathematics of Lorentz's transformation, but with different physical interpretations. This happened by introducing the concept of relative motion without referring to any ether. The theory is well- known today as the special theory of relativity ( STR ). Einstein's new theory caused a major shift at that time, because it changed the scientific thinking of many physicists into a non-ether context.

After the previous introduction, it will become prominent that the only ether which has survived historically and may get the acceptance scientifically is that medium which permeates all the universe with immobile and nonmechanical structure. The relationship between the ether and electromagnetic (EM) waves is simply the same relation between any wave and its carrying medium. The EM waves represent a disturbance which propagate through the ether, and the amplitude of this disturbance is represented physically by $(\mathbf{E}, \mathbf{B})$, the values of the EM fields, with no further specification. Through a two simple postulates, Sama aether Theory (SAT) demonstrates a new ether model, which is essentially built to give us a simple and direct answers for common questions that are usually asked in physics, like what is light? And where do gravity and matter waves come from?

\section{ETher as Multi-Rest Frames Medium}

\subsection{Defining the Rest Frame of Ether}

Initially, it is important to make a brief review on the traditional picture of the ether in the late $19^{\text {th }}$ century. An observer is said to be in rest frame, with respect to ether, when the velocity between the observer and the ether is classically equals to zero, therefore it represents an inertial frame. Any observer could simply "leaves" this rest frame by acting force on him, which would make him accelerates, and then be in motion with respect to ether.

Now as shown in ( Figure 1), for an observer located in a rest frame with respect to ether a propagating light signal will travel with the same speed in all spatial directions $(x, y, z)$. This happens because the speed between the observer and ether is zero. Mathematically, this means:

$$
\left|\vec{c}_{x}\right|=\left|\vec{c}_{y}\right|=\left|\vec{c}_{z}\right|=c
$$

Where $c$ is the speed of light. 


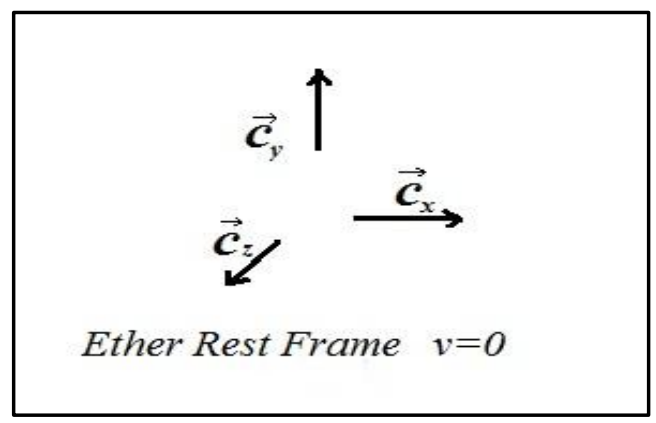

Fig1. When the velocity between the observer, and ether is classically equals to zero, the observer is said to be in rest frame with respect to ether, therefore light will propagate in all directions with the same speed.

Now when the observer leaves the rest frame by acceleration, he will be in motion with respect to ether, and this can be detected by the differences in the speed of light propagation according to the observer's speed and direction of motion with respect to ether. When the observer is moving with a speed $(v)$ in the same direction of light propagation, the speed of light will be reduced by $c^{\prime}=c-v$. Also the speed of light propagation will be increased if the observer is moving in the opposite direction as $c^{\prime}=c+v$. Many experiments were performed to detect such an effect (motion with respect to ether). In 1887, an experiment was done by two physicists named Albert Michelson and Edward Morley [4]. The experiment failed to detect any motion with respect to ether, and its failure was considered to be a major contradiction with the notion of ether.

\subsection{Existence of Multiple Rest Frames in Ether}

Now, let us take the following postulate about the ether:

"Existence of multiple equivalent rest frames in ether."

The meaning of this postulate is simply this, by saying " multiple "this means that there is more than one rest frame in ether. Therefore any observer can leave one rest frame, and gets into another rest frame, or in other words, the ability of different observers to occupy different rest frames in the same medium or ether.

Also by saying "equivalent" this means that all the rest frames in the ether are physically the same, or there is no any difference between them. Therefore, there will be no physical experiment that can differentiate one rest frame from another, and as a result of this, all the physical experiments will give the same results in all rest frames.

Now, let us take the following example for illustration. Assume the existence of two observers, observers (1) and (2) in a specific rest frame ( A) as illustrated in( Figure 2-a). Now, assume that observer (2) has the desire to leave the rest frame (A) and gets into another rest frame. As explained earlier, the only way that observer (2) can escape the rest frame is by applying a force on him, and in this situation he will be accelerating with respect to observer (1), (Figure 2-b).

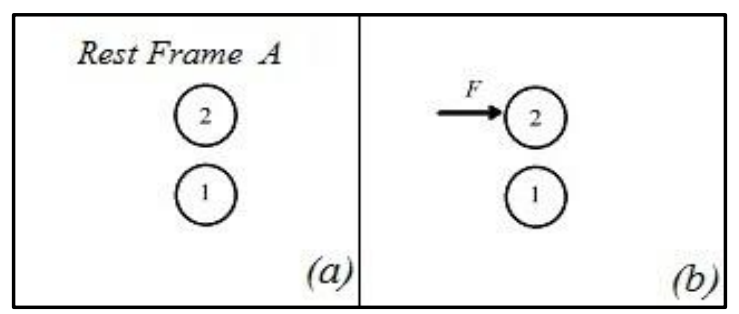

Fig2. (a) Both observers located in the same rest frame (A). (b) Observer (2) is leaving the rest frame by the acting force of magnitude $(F)$

According to the observer's (1) prospect, observer (2) can only be in three different states with respect to him, he is either be in his rest frame (A), or moving with a constant velocity, or accelerating. Since any rest frame represents an inertial frame, as explained earlier, this will mean that, if there is another rest frame in the ether other than (A), it should appear as a constant motion with respect to observer (1), because it is the only remaining inertial state.

Therefore if observer (2) wants to occupy a rest frame once again in ether, he simply has to stop the force that acting on him, and in this situation he will be moving with a constant velocity $\left(\mathbf{v}_{x}\right)$ with respect to observer (1). (Figure $\mathbf{3}$ ). 


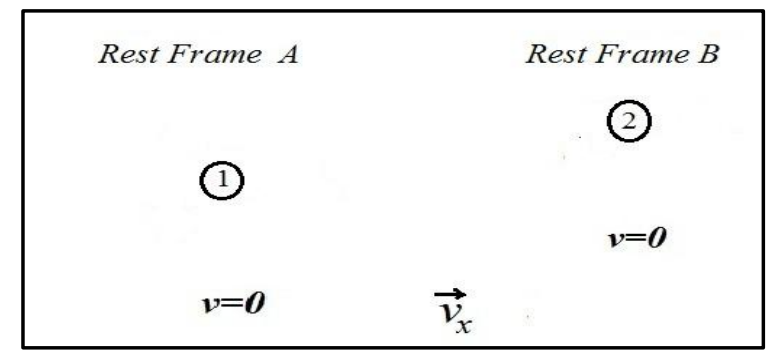

Fig3. Observer (2) now occupies a different rest frame in ether $(B)$, which means the velocity between him and ether is zero, but in the same time the speed $\left(v_{x}\right)$ between him and observer $(1)$ is more than zero.

Now observer (2), is in rest frame with respect to ether, which means that the velocity between him and the ether is zero, although he is moving with respect to observer(1) with a constant velocity (vx). Now, let us call it rest frame (B), and it differs from the rest frame (A) which is occupied by observer (1), since observer (2) is moving with a constant speed with respect to him.

Experimentally both observers can detect their states with respect to ether by sending a light signal in all three spatial directions. It will give the same result in Eq. (1), which means that both observers are in rest frames with respect to ether, although the existence of the constant speed (vx) between them.

Furthermore, since the two rest frames are physically equivalent, this means that there will be no physical experiment, that can differentiate rest frame (A) from rest frame (B), and as result of this all the physical experiments will give identical results in both rest frames if performed under similar physical conditions. This simply means that the physical laws will be the same in both rest frames.

Regarding the accelerating frames the speed of light will also be constant, although they are not rest frames in aether. The reason for this is given in section (3.3) when gravity is considered.

\subsection{Consequences Concerning the Existence of Multi-rest Frames Medium}

Sama aether represents multi-rest frames medium, and from this prospect, or point of view, the old traditional ether model will represent a uni-rest frame medium. The main difference between these two models of ether is that, the phenomenon of ether wind which results from the differences in the speed of light propagation cannot be detected in a multi-rest frames medium, but of course, it is detectable in the uni-rest frame medium, which was not proved to be existed experimentally. Therefore, the physical consequences of the first postulate, will be simply the consequences of constancy of speed of light as shown by in (1) for all observers in different rest frames in ether.

Let us take the following example about a light clock, owned by observer (1) existed in a rest frame (A). The clock composed of two arms, of the same length value $(L)$. The vertical arm is $\left(L_{y}\right)$, and the horizontal arm $\left(L_{x}\right)$, as illustrated in (Figure 4). The bulb sends a light signal in same direction of both arms, and then the signals are reflected by the mirrors, and reach the bulb again. The time interval, that is required for the light signal to cross the arm's length, and goes back is $(t)$, which is equals to $t=2 L_{x} / c=2 L_{y} / c$.

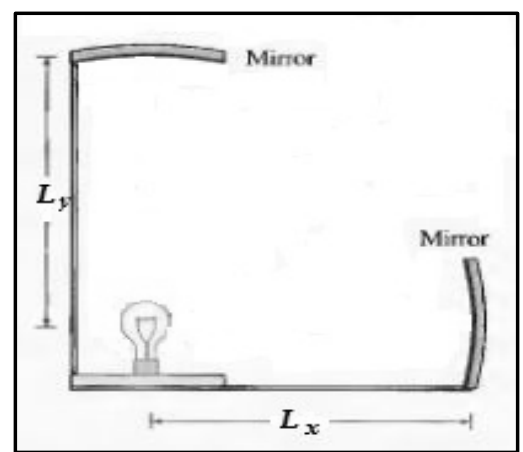

Fig4. A light clock. Light signal is sent from the light source, in the base along $L_{x}$ and $L_{y}$, and reflected back by the mirrors. 
Now consider another clock, which is identical to the previous clock. This second clock existed with another observer, observer (2), in a different rest frame. This means that it is moving with a constant speed $(v)$, with respect to observer (1).

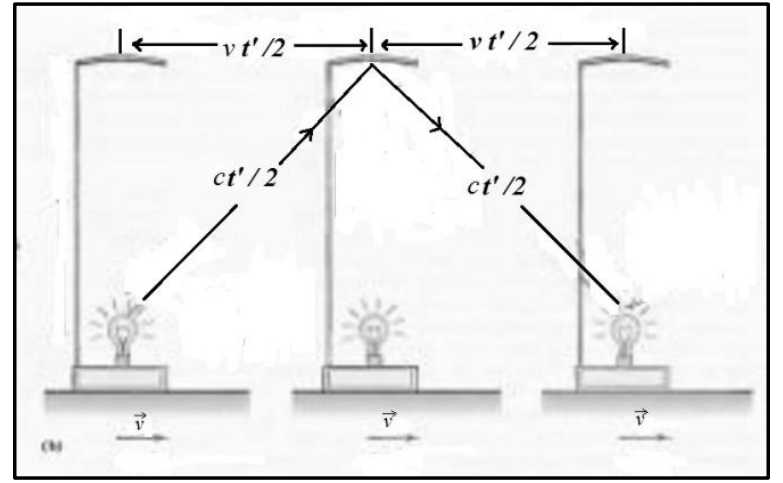

Fig5. The light clock is moving with speed (v) to the right. The vertical light signal will cross semi-triangular path with respect to observer (1).

Now observer (1) will detect the following measurements about observer's (2) clock. For the vertical arm, the light signal will cross a triangular path as illustrated in (Figure 5). The time interval which is required for the signal to travel to the mirror and come back $\left(t^{\prime}\right)$ where it is $\left(\frac{c t^{\prime}}{2}\right)^{2}=\left(\frac{v t^{\prime}}{2}\right)^{2}+\left(L_{y}^{\prime}\right)^{2}$. This equals to $t^{\prime}=\frac{2 L_{y}^{\prime}}{c \sqrt{1-v^{2} / c^{2}}}$.

Now, for the horizontal arm, since the clock is moving to the right, therefore when the signal travels outward, it will cross additional distance $\left(v t_{1}\right)$. Where $\left(t_{1}\right)$ is the time for outward trip. This gives $c t_{1}=L_{x}^{\prime}+v t_{1}$. For the return trip, the signal will cross a shorter path, since the bulb will be moving toward the signal. The time $\left(t_{2}\right)$, for the return trip will be $c t_{2}=L_{x}^{\prime}-v t_{2}$.

To get the value of $\left(t^{\prime}\right)$, we should add $\left(t_{1}\right)$, and $\left(t_{2}\right)$ as $t^{\prime}=t_{1}+t_{2}$ which gives $t^{\prime}=\frac{2 L_{x}^{\prime}}{c\left(1-v^{2} / c^{2}\right)}$. Now, by assuming that the value of the vertical arm $\left(L_{y}\right)$, will not be affected by motion, this means that $L_{y}=L^{\prime}$, and considering that both observers will detect the same speed for light propagation. This will give the following formulae:

$$
\begin{aligned}
& t^{\prime}=\frac{t}{\sqrt{1-v^{2} / c^{2}}} \\
& L_{x}^{\prime}=L_{x} \sqrt{1-v^{2} / c^{2}}
\end{aligned}
$$

Which shows that both spatial, and time interval will be distorted with motion. Time dilation with movement requires another physical quantity to be changed with motion. This is shown in the next example. Consider a clock composed of a particle, moving along the vertical axis $(\mathbf{y})$, with a constant speed $\left(v_{y}\right)$, which travels a specific displacement $(\Delta \mathbf{y})$ in a period of time $(T)$. For an observer which he in rest state with respect to this clock $(T)$ will be $T=\Delta y / v_{y}$. Also, the magnitude of the linear momentum of the particle in $(\mathbf{y})$, is given by $p_{y}=m v_{y}$.

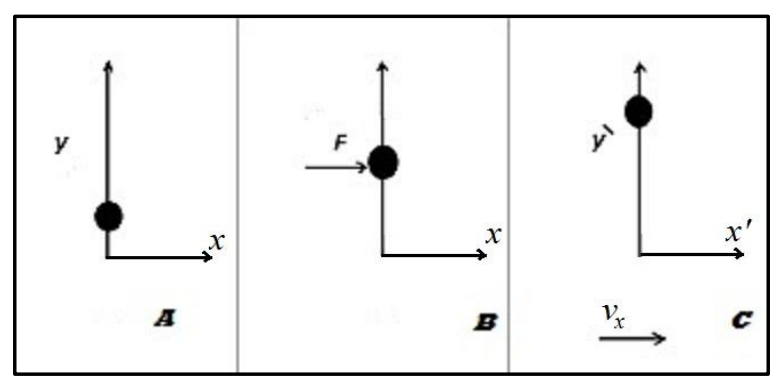

Fig6. (A) the particle is moving along the (y) axis. (B) An impulse is applied to the particle along the horizontal axis $(x) .(C)$ The particle now is moving in the $x$ direction with speed $\left(v_{x}\right)$.

Now, by applying an impulse with a force (F) on the particle on the horizontal axis ( $\mathbf{x}$ ). (Figure 6) The particle will acquire a velocity along the $(\mathbf{x})$ axis. Since the clock now is moving along the $(\mathbf{x})$ axis with velocity $\left(\mathbf{v}_{x}\right)$, the time 
interval $\left(T^{\prime}\right)$ that now will be required for the particle to pass the same $(\Delta \mathbf{y})$ interval will be a $T^{\prime}=\Delta y / v_{y}^{\prime}$. Since the impulse was in the same (x) direction, and not (y). This will mean that the magnitude of the momentum $\left(\mathbf{p}_{y}\right)$ will remain the same. This gives $p_{y}{ }^{\prime}=p_{y}$. Now, the relation between $\left(T^{\prime}\right)$, and $(T)$ is given by the time dilatation formula:

$$
T^{\prime}=\frac{T}{\sqrt{1-v_{x}^{2} / c^{2}}}
$$

This means that the speed $\left(v_{y}{ }^{\prime}\right)$ after the impulse is $v_{y}^{\prime}=v_{y} \sqrt{1-v_{x}^{2} / c^{2}}$. But, the magnitude of the momentum $\left(\mathbf{p}_{y}\right)$ is conserved $\vec{p}_{y}=m^{\prime} \vec{v}_{y}^{\prime}=m \vec{v}_{y}$. This requires that the value of mass $\left(m^{\prime}\right)$ is changed to $m^{\prime}=\frac{m}{\sqrt{1-v_{x}^{2} / c^{2}}}$.

Now, let us take a very small value of $\left(v_{y}\right)$, where its physical value becomes negligible. This will change the previous equation to :

$$
m^{\prime}=\frac{m_{0}}{\sqrt{1-v_{x}^{2} / c^{2}}}
$$

Where $\left(m_{0}\right)$ is rest mass of the particle.

This is will change the value of the momentum of any moving body, with a rest mass $\left(m_{0}\right)$ according to the equation:

$$
\vec{p}=m \vec{v}=\frac{m_{0} \vec{v}}{\sqrt{1-v^{2} / c^{2}}}
$$

\section{THE MULTIPLICITY OF ENERGY FORMS IN ETHER}

Energy represents a scalar physical quantity, that an object or physical system posses. It has many forms, like kinetic energy and potential energy. The energy's forms are deeply connected in nature, and can transform from one form to another. In modern physics, the quantum behavior of light was introduced by A. Einstein in 1905 to explain the photoelectric effect. Later on, in the 1940s, a quantum theory for the EM phenomena was developed, which is the quantum electrodynamics theory. Now, here we will use a simple postulate to make a connection between the nature of light and some other physical phenomena as will be discussed later. Therefore, let us take the following assumption about ether:

\section{"The kinetic energy, and the potential energy are the only forms of energy for ether's waves."}

The meaning of this postulate is simply this, the kinetic energy, represents a mechanical form of energy, which means that, it is always carried on particles or quanta. Therefore, and as a result of this, the energy of the ether's waves is always carried on particles or quanta. Now, if these quanta are free quanta, which mean that they are not affected by any conservative force, here the kinetic energy will be the only form of energy for the ether's wave, but if these quanta are affected by a conservative force, the quanta will acquire an additional form of energy which is the potential energy. Later on, we will see that the quantum has an energy of $(h v)$, which means that it could be described by a wave function in identical manner to that of quantum mechanics. Which means also in this situation that the quantum will represent the EM field itself, or in other words the EM field will be a matter by itself represented by particles.

\subsection{The Nature of Light in SAT}

The physical importance of the previous postulate is that the wave theory in physics does not forbid a wave from carrying its energy in the kinetic, and the potential forms just like sound waves or all the other mechanical waves. The major difference between the electromagnetic (EM) waves and the mechanical waves is simply the amplitude of the wave.

The amplitude of the sound wave (or in general the mechanical waves) contains a velocity vector which results in a movement associated with the amplitude itself, and since any particle should be in motion to possess a kinetic energy, the particles in the sound waves will move according to the amplitude's velocity vector back and forth (Figure 7). This in turn, results in a roughly "continuous" distribution of energy along the wave front. This concept is hold in all mechanical waves. 


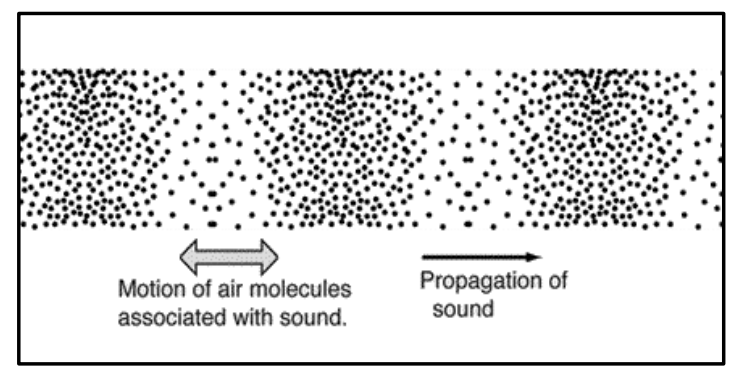

Fig7. Sound waves in air. There are two types of motions in any mechanical wave. The amplitude's motion and the propagation motion.

On the other hand, concerning the electromagnetic waves, or generally the ether's waves, as will be seen later. The amplitude's motion does not exist, and waves contain only one direction of the velocity vector, which points toward the direction of propagation, as illustrated in (Figure 8). Again, since the particles or quanta which carry the wave's energy should be in motion to possess kinetic energy, the only way that will be possible for these particles or quanta to move, is to move along the direction of the propagation velocity vector. Therefore, technically when the wave's energy is detected by a detector, it will be detected in localized discrete spots.

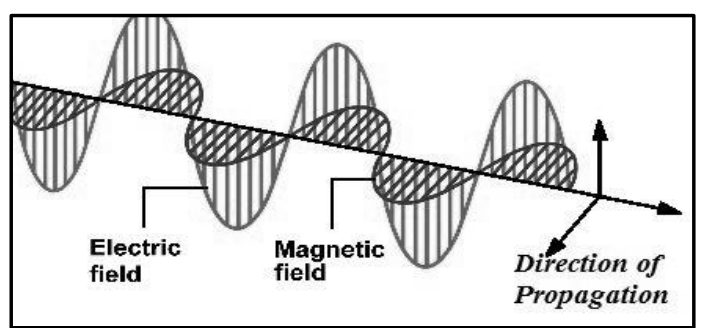

Fig8. The EM waves. There is only one direction of movement, which is the propagation direction

Regarding the nature of light, a common question is usually asked. What is light? This question is simply answered from the prospect of the second postulate as this, light is a wave that carries kinetic energy. The physical value of this definition is that, the wave theory does not forbid a wave from carrying its energy in the mechanical (kinetic and potential) forms, just like saying, sound is a wave that carries a kinetic energy, or water wave is a wave that carries its energy in the kinetic form, which in the three situations (Light, water and sound waves) will be carried on particles. As a result of this, there will be no need to assume that light has a dual nature (wave-particle duality), since the wave theory alone can account its physical behavior from this prospect.

Light propagates at speed considered to be $c$ and this requires that particles or quanta which carry the wave's energy to be massless in order to move with the same speed. As will be demonstrated in section (3.3), the total energy of "free" massless particle is completely kinetic, due to absence of rest energy. Now, let us ask ourselves a simple question. What will happen if that light's particle has a rest energy and therefore rest mass, but for unknown reason it is not detectable experimentally? This will represent a clear violation to the second postulate, since the rest energy is neither kinetic nor potential energy and therefore the validity of the second postulate will be questionable. A solution to this problem will be considered later in this paper.

The second postulate of ether theory is best understood as a reflection of a deeper reality existed in the nature. The electric field around an electrically charged particle represents a vector quantity that spreads continuously throughout space. The electric field does carry energy, but what form of energy? This is the important question that should be asked. Since energy comes in many forms, the ancient physicists simply assumed that the electric field has its own form of energy which is the electric field energy. The electric field energy represents a form of energy that spreads continuously with field itself. From here, when the value of the electric field changes with time in a specific point in space, and propagates as electromagnetic wave, the energy of the field (wave's energy) will spread continuously along the wave front, and therefore will be detected continuously by a detector. Certainly, this is something that was not proved to be existed experimentally.

The electric (magnetic) field form of energy does not exist in nature, therefore the electric (magnetic) field lacks its own form of energy, and instead, it carries its energy in the kinetic form (which means particles), although the field itself spreads continuously in space. Therefore, the number of energy forms that are really existed in nature is fewer 


\section{American Research Journal of Physics, Volume 1, Issue 1, 2015} ISSN 2380-5714

than was assumed. A simple result of this, is that the electromagnetic interactions between the charged particle, will not occur instantaneously, since it depends on the field's energy, which in turn carried by quanta that moves with a specific speed, which assumed to be $(c)$, the speed of light.

Now, let us consider a classical example to calculate the energy of the light's quanta from basic principles. A monochromatic electromagnetic radiation is emitted from a source. The radiation will spread approximately in a spherical pattern. (Figure9).

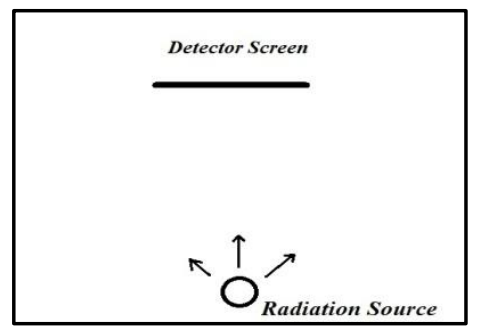

Fig9. The intensity of the radiation will be measured by a detector located away from the source.

Since the radiation will spread in a spherical pattern, a scalar spherical wave equation for the electric field (E), will be used[8] $\mathrm{E}=\frac{\mathrm{E}_{\circ}}{r} e^{i(\omega t-k r)}$ Where $(\omega)$ is angular frequency, (r) corresponds to the distance from the source, and (k) is the wave number. Now, by taking the real part of this equation, since it has the physical meaning, this gives:

$$
\mathrm{E}=\frac{\mathrm{E}_{\circ}}{r} \cos (\omega t-k r)
$$

Since the energy of the radiation is carried with same speed of the waves, which assumed to be $(c)$. This result in that the quanta that carry the wave's energy will be considered to be massless in order to move with the same speed $(c)$. The energy $(E)$ of a massless quantum is given by the formula $E=p c$ where $(p)$ is the magnitude of the linear momentum of the quantum. Therefore the intensity $(I)$ of the radiation at the detector will be:

$$
I=n p c
$$

Where $(n)$ is the number of quanta that reaches a unit area, in a unit time. The radiation will stop when the magnitude of the electric field (E), that changes with time is equals to zero. From equation (6), this means:

$$
\frac{\partial^{2} \mathrm{E}}{\partial t^{2}}=-\omega^{2} \mathrm{E}=0
$$

Also, when the radiation stops, the intensity $(I)$, at the detector will be zero. This means that when Eq. (8), changes to zero, Eq. (7), will also change to zero, which represents a function between the variables of the two equations as $n E=f(\omega, E)$. Furthermore, since the wave spreads spherically, the value of $(I)$ will decrease as you go far from the source, because the energy of the wave spreads over a larger spherical surface area( drops by $\left.1 / r^{2}\right)$.

Also, since the linear momentum in the equation $(E=p c)$ is a conserved linear quantity, this indicates that the drop in $(I)$ is just result from $(n)$, and since $(\omega)$ is constant along $(r)$, this indicates that $(n)$ does not change with $(\omega)$, which gives $n \neq f(\omega)$, but the value of Eq. (8) should be zero, when $(n)$ is zero, this indicates that $n=f(\mathrm{E})$.

Again, the value of intensity $(n E)$ should be zero, when Eq. (8) is equals to zero, but since $(n)$ is not function in $(\omega)$, this means that $(E$ or $p c)$ changes to zero when $(\omega)$ is zero, which gives $E=f(\omega)$. Since $(c=\omega / k)$, this gives $E=$ $p c=p \omega / k$.

Since $(E)$ is only a function in $(\omega)$, this means that $(p / k)$ represents a constant value $(u)$ for $(E)$, which gives:

$$
E=u \omega
$$

This constant value $(u)$ is indeed the well-known reduced Planck's constant $(\hbar)$.

The importance of the previous example is that it demonstrates that the possibility to derive the formula in (9), from a simple calculations based on the classical electrodynamics, and the postulates of ether theory.

\subsection{Matter Waves, what are they?}

By considering a relevant point. As discussed earlier, the EM wave represents a disturbance that propagates through the ether, where $(\mathbf{E}, \mathbf{B})$ are the values of the amplitude of this disturbance. Now, let us assume that there is another 


\section{American Research Journal of Physics, Volume 1, Issue 1, 2015}

ISSN 2380-5714

disturbance which propagates through the ether as a wave, but with different amplitude's value (S), where (S) represents the magnitude of the field measured with arbitrary units $(s)$. The EM wave and the (S) wave share the fact that they are both a propagating disturbances in the same physical entity, which is the ether itself, but each one represents a different physical value of the amplitude.

Since (S) represents a wave in ether, the second postulate of SAT, should be applied for it, in the same way as for light, which means that its energy will be carried on quanta, and since the wave of $(S)$ is a field wave, the quanta will be moving according to the direction of the propagation vector, just as described earlier for light. A difference will be made, which is the quantum of(S) will be considered to have a detectable rest mass. Initially, this represents a clear violation to the second postulate, since the rest masses of these quanta will have an equivalent value of rest energy, which is neither a kinetic energy nor a potential energy, and will contribute to the wave's energy. We will consider this violation later, but now let us ignore this contradiction.

Now, let us consider a hypothetical experiment, which is the double-slit experiment for the wave (S). As illustrated. ( Figure 10).

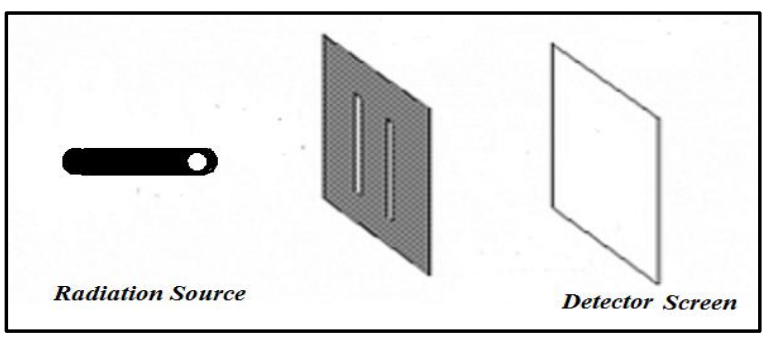

Fig10. A radiation of $(S)$ is emitted from imaginary source to pass through a double-silt barrier to be detected after by a screen

A monochromatic radiation of(S) is emitted from the imaginary source, and passes through the double- silt. An interference pattern is expected to occur at the detector screen, when the radiation is emitted over a period of time, as shown in ( Figure 10).

The source will radiate the wave (S), and since the quanta which carry the wave's energy have a detectable rest mass, it will move with a speed less than that of light. Now, when the quanta hit the screen, it will be detected as localized spots in the screen detector. After a period of time a diffraction pattern is expected to be detected on the screen.

Because the field (S) propagates as a wave, its value will change with time and place. The wave's energy will depend on $\left(S^{2}\right)$, which represents the average of a complete cycle of the square of the instantaneous magnitude of (S) field in a specific place, just like EM radiation.

The area on the screen where a constructive interference occurs, the average value of $\left(S^{2}\right)$ will be expected to be the maximum; therefore it will receive the maximum number of quanta per unit time (maximum intensity). On the other hand, the area where a destructive interference occurs, the average value of $\left(\mathrm{S}^{2}\right)$ will be expected to be the minimum, therefore, it will receive the minimum number of quanta per unit time ( minimum intensity). ( Figure 11).

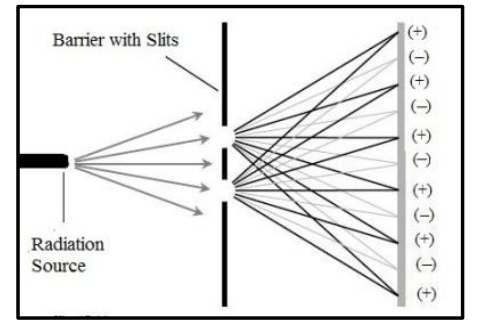

Fig11. Double slit experiment. Areas of constructive interference (+), and areas of destructive interference( -- ).

This experiment illustrates the possibility to describe (S) waves by a completely different manner. Instead of using the value of field (S) to describe the amplitude of the wave, another value will be used which is ( $\left.\psi_{\mathrm{s}}\right)$. The physical meaning of this description is that the probability of experimentally detecting the quantum of the field $(\mathrm{S})$ in specific point in space and time $(t)$ is proportional to $\left|\psi_{\mathrm{s}}\right|^{2}$ at that point and in $(t)$. Therefore it represents a probability density. This interpretation is similar with that of EM wave's double silts experiment [9]. 


\section{American Research Journal of Physics, Volume 1, Issue 1, 2015 ISSN 2380-5714}

Now, let us consider another disturbance propagates through the ether with a different amplitude value, field (G). Again since it represents a wave in ether, therefore the second postulate is applied. As a result of this, its kinetic energy will be carried on quanta just like EM and (S) waves. Also, the conclusion of the previous double-slit experiment is applicable for $(\mathrm{G})$, this means that the value $\left(\psi_{\mathrm{G}}\right)$ could describe the amplitude of the wave instead of the field $(\mathrm{G})$. Again $\left|\psi_{\mathrm{G}}\right|^{2}$ will determine the probability of finding the quantum of the field $(\mathrm{G})$ in a specific point in space and time.

By using the dimensional analysis, the physical dimensions of both $\left|\psi_{\mathrm{G}}\right|^{2}$, and $\left|\psi_{\mathrm{s}}\right|^{2}$ will be the same, since both represent a probability density. Therefore a single value will be used to describe (S), and (G) or any other wave's amplitude in ether, which is $(\psi)$, but when this value is used, the only way that will be possible to differentiate between the wave field (S), and the wave field (G) is by saying that the value of $|\psi|^{2}$ is associated with the probability of detecting the quantum or particle (S) in specific point in space and time, when describing the wave field (S).

Now, when the description is about the wave field $(G),|\psi|^{2}$ will be associated with the probability of detecting the quantum or particle $(G)$ in a specific point in space and time. Therefore, if the wave field $(G)$ is associated with a positive electric charge, the particle or quantum $(\mathrm{G})$ will be considered a positively charged particle, and so on for the other physical properties. Therefore, it is physically equivalent to matter waves.

The general definition of a wave is that it represents a disturbance which propagates from one point to another. Therefore, the wave's energy is simply represents the energy of the propagating disturbance itself. In the case of ether, it will represent the quantum's energy which carries the wave's energy, which will be taken as $\left(E_{\mathrm{d}}\right)$. Also, the general definition of the wave's frequency is that it represents the number of disturbances that pass through a specific point in unit time. For ether's waves it will be taken as $\left(v_{d}\right)$. From the previous Equation (9). We find that there is a constant value between the frequency, and the energy of the ether's waves as:

$$
2 \pi \hbar=\frac{E_{d}}{v_{d}}
$$

Therefore, this equation will be valued for any disturbance propagating in ether, since $\left(E_{d}\right)$, and $\left(v_{d}\right)$ represent the energy and the frequency of the disturbance, regardless of its amplitude value ( whether it is the EM fields, or the hypothetical (G), and (S) fields).

After all the previous discussion, it will become obvious that the wave-particle duality does not exist in SAT. What is really exists are the ether's waves which share the same medium, but differ in the amplitude's value, and carry kinetic and potential energy, with no any contradiction with the wave theory as explained earlier.

\subsection{The Origin of Gravity}

We will start this section by defining the ordinary kinetic energy for a particle. When a force of value $(\mathbf{F})$ is applied on a particle with a rest mass $\left(m_{0}\right)$, the kinetic energy $(K E)$ will represent the work $(W)$ which was done by this force, as $K E=W=\int_{0}^{\vec{s}} \vec{F} . d \vec{s}=\int_{0}^{p} v d p \quad$ where (s) is the displacement acted upon the force (F). Now, let us recall the equation of a linear momentum which is $\vec{p}=m \vec{v}=\frac{m_{0} \vec{v}}{\sqrt{1-v^{2} / c^{2}}}$. When this formula is applied in the equation of the kinetic energy above, this will give:

$$
K E=\frac{m_{0} c^{2}}{\sqrt{1-v^{2} / c^{2}}}-m_{0} c^{2}
$$

By defining the total energy $(E)$ of the particle as the sum of the rest energy $\left(E_{0}\right)$ and kinetic energy:

$$
E=K E+E \text { 。 }
$$

And by combining (10) and (11), another equation is obtained which is $\quad\left(K E+E_{\circ}\right)^{2}=E^{2}=(p c)^{2}+E_{\circ}^{2}$. Now, when considering a massless particle, which lacks a rest mass, and therefore rest energy, the previous equation will give $K E^{2}=E^{2}=(p c)^{2}$, and by taking the positive root of this equation, this gives:

$$
K E_{2}=E=p c
$$

The kinetic energy which appear in this equation (12) is different from the kinetic energy in equation (10) from two prospects: 
The kinetic energy in (12), is not a function in the particle's speed as does (10), because the particle is massless, and moves with a constant speed considered to be $(c)$. Therefore it cannot be defined as the work done by a specific force, in certain displacement just like the kinetic energy in equation (10).

$>$ The kinetic energy in (12), represents the total energy of the particle, in contrast with the kinetic energy in (10) which represents only the additional part of energy which is acquired from motion( besides rest energy).

Regarding these two differences the kinetic energy in (12) it is not the same as that in (10). Therefore, the simplest way to deal with the kinetic energy in (12) is to consider it as another or second form $\left(K E_{2}\right)$ of kinetic energy. Therefore, the total energy of "free" mass less particle is completely kinetic $K E_{2}=p c$.

Now, let us recall the second postulate of ether, which states that, kinetic and potential energies are the only forms of energy for ether's waves. The potential energy represents the effect of the conservative forces which acting on the particle. Therefore, from this postulate when a particle is free (no action of any force), the kinetic energy will be the only form of energy. Obviously this is cannot be true, since particles that carry wave's energy are not always considered to be massless, which means that when they have rest mass and therefore rest energy, it will contribute to the wave's energy, which is neither kinetic nor potential energies. This represents a contradiction to the second postulate.

To resolve this problem, if we hold the second postulate as a valued principle, the rest energy should be considered as a form of kinetic energy or simply a third form of kinetic energy ( $\left.K E_{3}\right)$, and this is the step that will be taken here, because at this situation, the total energy of any free particle will be always kinetic $\left(K E+K E_{3}\right)$. The general notion or idea about kinetic energy is obtained from (10), which is that it represents an amount of particle's energy that is acquired from motion. Therefore, defining the rest energy $\left(E_{0}\right)$ as a kinetic energy is done by defining it as a function in some kind of motion or speed $\left(v_{0}\right)$ :

$$
E_{0} \equiv K E_{3}=f\left(v_{0}\right)
$$

Where $E_{0}=0$ when $v_{0}=0$. Therefore the value of $\left(\mathrm{E}_{0}\right)$ will depend on $\left(\mathrm{v}_{0}\right)$. The main problem with this step is that the particle is at rest, which means that its speed and velocity equal zero, but this is not completely true, because the rest state by itself permits a particle to move in a specific type of linear motion. For the sake of illustration, a classical point particle is taken to be in rest, and since the particle is classical, it will has a known position which is the origin, and a known velocity which is zero(v=0), as shown in (Figure12).

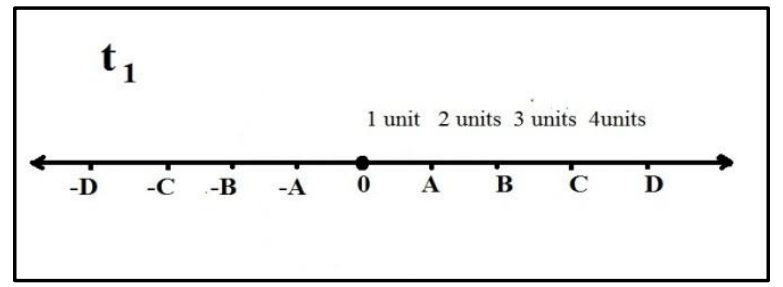

Fig12. At the moment $\left(t_{1}\right)$, the point particle is located in the origin.

Now, let us assume that at the moment $\left(t_{l}\right)$ the particle has a velocity of the magnitude $\left(v_{0}\right)$ and its direction is to right. At the moment $\left(t_{2}\right)$, the particle position is changed to (A), due to its motion. Now, if the particle in the same time is moving toward the opposite direction with the same magnitude $\left(v_{0}\right)$, this will mean that at $\left(t_{2}\right)$, the particle's position is changed to (-A ) also. Mathematically, this type of motion, the rest motion is expressed by a simple equation:

$$
\vec{v}_{0}+\left(-\vec{v}_{0}\right)=0=\vec{v}
$$

Where $(\vec{v})$ is the particle's velocity, and $\left(\vec{v}_{0}\right)$ is the particle's rest velocity. This means that the particle's velocity is zero, but its rest velocity is not zero. From (14), the particle's displacement at any moment will represent the result from moving into two opposite directions with the same magnitude at the same time $\Delta t \vec{v}_{0}+\left(-\Delta t \vec{v}_{0}\right)=0$.

Now, if this type of motion is really happening, this will mean that at $\left(t_{2}\right)$ the particle is in position (A) which is separated only by one unit from position (B) and two units from position $(\mathbf{C})$ and so on. Also at $\left(\mathrm{t}_{2}\right)$ the particle is in position $(-\mathbf{A})$, which is separated by one unit from position $(-\mathbf{B})$. Therefore at $\left(\mathrm{t}_{2}\right)$, both positions $(\mathbf{B})$ and $(-\mathbf{B})$, will be separated by one unite from the particle in opposite direction. (Figure 13). 
Finally, the particle at $\left(\mathrm{t}_{2}\right)$ occupies position $(\mathbf{A})$, and position $(-\mathbf{A})$. Both are equal to one position, the origin $\vec{A}+(-\vec{A})=0$ which is the same initial position occupied by the particle at $\left(\mathrm{t}_{1}\right)$.

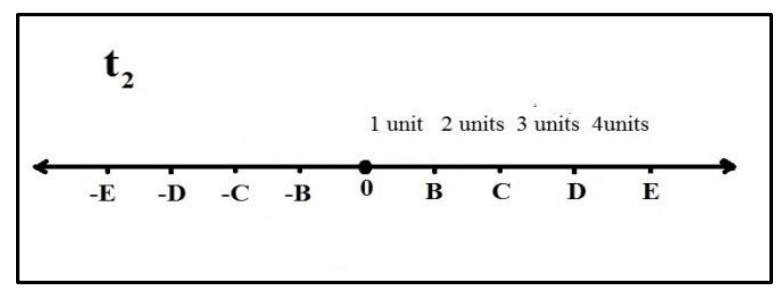

Fig13. At the moment $\left(t_{2}\right)$, the point particle is located in the same position, the origin, but the values of the surrounding positions are changed.

Therefore, a simple conclusion about the rest motion is this "The position of the particle does not change at any moment, since the particle is at rest, but what is really change is the value of positions surrounding the particle with the same magnitude but with opposite direction". (Figure 13).

The previous description of the rest motion is just a primitive or initial description; therefore it is not applicable in nature by its current form. To get an accurate description of the rest motion, let us consider a body with relatively huge mass $(M)$. The body will be chosen to be:

Static, so that the body will has only one type of motion, which is the rest motion, and any other type of motion is neglected.

> Spherically symmetric with radius ( $\mathrm{r} 0)$, so that the value of the rest motion (v0+ (-v0)), will be the same, regardless of the spatial direction which is chosen $(\mathrm{x}, \mathrm{y}, \mathrm{z})$.

Now, let us look at the following hypothetical physical equation between two variables $(A, J)$ in $A=\frac{J P}{Y}$. The relation between the variables will be the same in this equation whether you consider that $(A)$ is function in $(J)$, or $(J)$ is function in $(A)$, since the dimensional analysis will be the same in both cases, but it will only change the definition of the function. By getting back to our problem, a similar step will be taken in order to simplify the calculations by assuming that $v_{0}=f\left(E_{0}\right)$ in comparison with equation (13).

The effects of the rest motion will be considered outside the body only for the sake of simplification. Now, let us take two observers. Observer (1) is at the surface of the body. Observer (2) is the observer which is not affected by the rest motion of the body, therefore he will represent the stationary observer. For the moment, the physical properties of the rest motion are unknown, therefore, the position of the stationary observer (2) will be considered unknown.

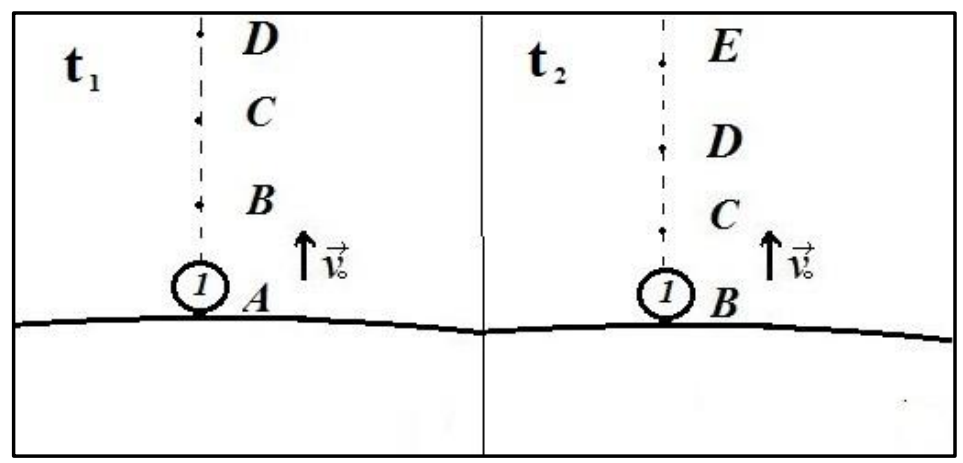

Fig14. Observer (1) is located on the surface of the spherical body, in contact with it. Therefore, he is moving with the body's surface by the same rest speed $\left(v_{0}\right)$.

Because the body is symmetrically spherical, the rest speed will be the same at any point on the surface. Observer (2) detects the rest motion of observer (1) between two moments $\left(t_{1}\right)$ and $\left(t_{2}\right)$, by changing in his position between (A) and (B).

From (13), the rest energy of the body depends on its rest speed $\left(v_{0}\right)$. Therefore when the magnitude of the rest speed equals zero, the rest energy, and therefore the rest mass $(M)$ of the body should also be zero (Figure 15). 


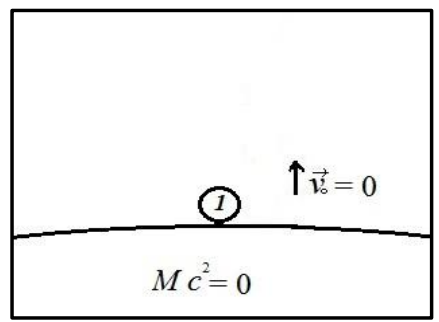

Fig15

This situation in (Figure 15), is physically identical to the following situation. Consider that observer (1) is located in free space-time, with no any detectable mass surrounding him. In this situation the value of the rest speed that affects observer (1) is zero due to absence of any body surrounding him $(M=0)$,( Figure16).

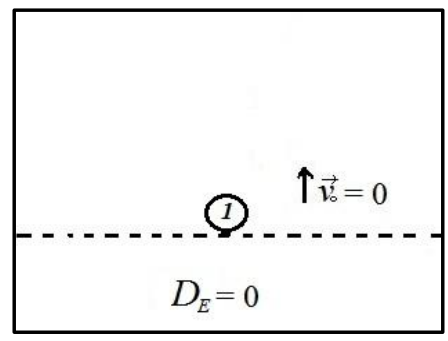

Fig16

By looking at (Figure16), the energy density (DE) under the imaginary line ( dashed line) below observer (1) in free space-time is classically considered to be zero, but this will also mean that observer (1) is located on a surface of a spherical body with a nonzero value of rest energy (E0), but its center is located in infinite point, because at this situation the energy density below the dashed line will also be zero. This occurs because the relation between the two is $\mathbf{D}_{\mathbf{E}}=\mathbf{E}_{\mathbf{0}} / \frac{4}{3} \mathbf{\pi} \mathbf{r}_{\mathbf{0}}^{3}$. Therefore, when the radius of the spherical body goes to infinite value $(\mathrm{r} 0=\infty)$, the density will be zero, which means free space-time.

Therefore, if a body has a nonzero value of rest energy, but its energy is distributed over infinite spatial volume, the value of $v_{0}$ will be zero, because this situation is identical to that in (Figure16). From here, the following relation is hold $v_{0}=f\left(D_{E}\right)$ where $v_{0}=0$, when $D_{E}=0$.

When considering spherical volumes only, the rest energy density $\left(D_{E}\right)$ will depend on two variables, which are the amount of rest energy $\left(E_{0}\right)$, and $(r)$, which represents the radius of the spherical volume which is considered. Therefore, the previous equation $v_{0}=f\left(D_{E}\right)$ could be written as $v_{0}=f\left(E_{0}, r\right)$. Therefore, at the surface of the body where observer (1) is located, the radius of the volume which is considered is simply the body's radius $\left(r_{0}\right)$, where the value of the rest speed is $\left(v_{0}\right)$. But in any other value of $(r)$ away from the body's surface, the value of $\left(v_{0}\right)$ will change, and this will lead us to the following equivalence.

The Equivalence is simply this, by looking at (Figure17), let us consider another observer, observer (3) which located in the first floor in the tower as shown in the figure. From his prospect, he can consider himself located on imaginary surface (dashed line) of a body with the same rest energy $\left(E_{0}\right)$, but with a radius $\left(r_{3}\right)$, and since the value of $\left(v_{0}\right)$ changes with the radius, it will change to $\left(v_{3}\right)$. The same is applied for observer (4), how is located in the second floor. This equivalence is hold because the energy density of body decreases as you go far from the body by $\left(1 / r^{3}\right)$, and this by itself causes a drop in the value of rest speed, which means $v_{0}>v_{3}>v_{4}$.

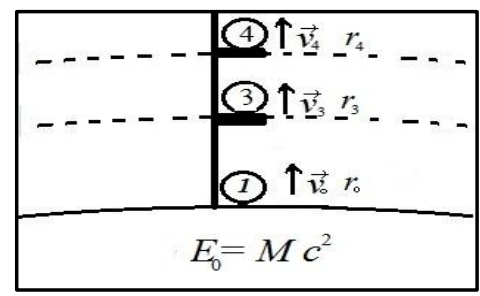

Fig17 


\section{American Research Journal of Physics, Volume 1, Issue 1, 2015 ISSN 2380-5714}

Therefore, the previous observer (2) which represents the stationary observer that is not affected by the rest motion of the body should be located away at infinite distance. Because at this distance the value of $(v)$ is zero, because the energy density is zero $r=\infty$. This represents an imaginary location, since $(\infty)$ is undefined mathematical quantity.

By getting back to observer (1), and observer (2). Let us consider that both observers (1 and 2) have clocks in their locations. Since observer (1), and his clock are moving with respect to the "stationary" observer (2) with a constant speed value $v_{0}$ (Figure14). By using the time dilatation formula, this result:

$$
\Delta t=\frac{\Delta t^{\prime}}{\sqrt{1-v_{0}^{2} / c^{2}}}
$$

Where $\left(\Delta \mathrm{t}^{\prime}\right)$ is time interval for observer $(1),\left(v_{0}\right)$ is the rest speed of the body, and $(\Delta t)$ is time interval for observer (2). Also, due to motion, any length interval should be contracted along the direction of movement ( radial direction toward the body's center). By using the formula of the length contraction:

$$
d r=d r^{\prime} \sqrt{1-v_{0}^{2} / c^{2}}
$$

Where $\left(d r^{\prime}\right)$ is a small length interval for observer (1), and $(d r)$ is the same length at observer (2).

Now, assume that observer (2) has a test particle with a rest mass $\left(m_{0}\right)$ which is negligible compared with $(M)$. The particle's energy at observer's (2) location is $m_{0} c^{2}$. When the same particle is located with observer (1) on the body surface, it will has additional kinetic energy, because it will be moving with the observer (1) with the same rest $\operatorname{speed}\left(v_{0}\right)$ :

$$
K E_{1}=\frac{m_{0} c^{2}}{\sqrt{1-v_{0}^{2} / c^{2}}}-m_{0} c^{2}
$$

For a specific body (M ), the value (v0) is only a function in (r). Therefore, (v0) is the only variable in the right side of the above equation for a specific test particle (m0). This will mean that the kinetic energy of the test particle in (17) will also change with (r) as $\mathrm{KE}_{1}=\mathrm{f}(\mathrm{r})$. Since the kinetic energy represents the work (W) done by a force (F) for a specific displacement value as $d K E=d W=\vec{F} . d \vec{r}=F d r$ where (r) is the magnitude of the radial position vector $(r)$ from the center of the body. Therefore, the kinetic energy will be equivalent to $\mathrm{KE}_{1}=\int_{\infty}^{\mathrm{r}_{0}} \mathrm{~F} \mathrm{dr}$. This will equal to:

$$
\mathrm{KE}_{1}=\frac{\mathrm{m}_{0} \mathrm{c}^{2}}{\sqrt{1-\mathrm{v}_{\mathrm{r}}^{2} / \mathrm{c}^{2}}}-\mathrm{m}_{0} \mathrm{c}^{2}
$$

where ( $\mathrm{v} r$ ) is speed of the test particle along ( $\mathrm{r}$ ) detected by observer (1) when the test particle reaches the body surface.

Since the kinetic energy in (18) represents the same kinetic energy in (17), therefore, by comparing them, this gives:

$$
\mathrm{v}_{\mathrm{r}}=\mathrm{v}_{0}
$$

If the test particle loses its kinetic energy in (18), when it reaches the surface of the body, it will be restricted on the body surface by a potential energy (PE) of the same magnitude of its previous kinetic energy:

$$
\mathrm{PE}=-\left(\frac{\mathrm{m}_{0} \mathrm{c}^{2}}{\sqrt{1-\mathrm{v}_{\mathrm{r}}^{2} / \mathrm{c}^{2}}}-\mathrm{m}_{0} \mathrm{c}^{2}\right)
$$

Therefore, from the previous equation, the value of (vr) will be the same speed needed for the test particle to escape from the body surface along the direction of $(r)$.

Now, if all the previous physical effects of the rest motion of the body $(M)$ is considered to be equivalent to the gravitational effects around it. This will simply mean that $\left(\mathbf{v}_{r}\right)$ will represent the same gravitational escape velocity, which its magnitude could be obtained simply from Newton's law of gravitation [10] $v_{r}^{2}=2 G M / r_{0}$.From (19), the value of $\left(v_{0}\right)$ will be the same as the previous equation $v_{0}^{2}=2 G M / r_{0}$.

By applying the value of $\left(v_{0}\right)$ in (15), and (16):

$$
\Delta t=\frac{\Delta t^{\prime}}{\sqrt{1-\frac{2 G M}{r_{0} c^{2}}}}
$$




$$
d r=d r^{\prime} \sqrt{1-\frac{2 G M}{r_{0} c^{2}}}
$$

The previous two equations indicate that the speed of light in accelerating frame is also a constant value due to the effect of time dilatation and length contraction. Also by substituting the value of $\left(v_{r}\right)$ in $(20)$. The magnitude of the force which affects the test particle is easily calculated $F=-\frac{d P E}{d r}$ as :

$$
F=G \frac{M m_{0}}{r_{0}^{2}}\left(1-\frac{2 G M}{r_{0} c^{2}}\right)^{-\frac{3}{2}}
$$

Now, the kinetic energy of the rest motion $\left(K E_{3}\right)$ for the body $(M)$ and specific radius $\left(r_{0}\right)$ is simply calculated from $v_{0}^{2}=\frac{2 G M}{r_{0}}$ Since the rest energy of the same body is $K E_{3}=E_{0}=M c^{2}$. By combining these two equations, this gives:

$K E_{3}\left(v_{0}\right)=\frac{r_{0} c^{2} v_{0}^{2}}{2 G}$

This represents the third form of kinetic energy for a symmetrically spherical, static body, with a specific radius $\left(r_{0}\right)$ which satisfies (13).

It is important to note that acceleration which appears in (23) breaks the symmetry of the relative motion of the rest speed $\left(v_{0}\right)$ between observers (1) and (2). Because the situation is similar to that of the famous twins paradox[10]. From here the space-time curvature which is demonstrated by (21), and (22) will not be a reciprocal effect between the two observers. Also the description of gravity was completely classical, but a glimpse of what direction that should be taken to build a quantum theory of gravity was considered in a previous paper [11].

Also, it is important to note that the gravitational time dilatation formula in (21), and the gravitational force in (23) represent the same physical effect. To explain this, let us consider a beam of monochromatic EM waves passing along the vector $(\mathbf{r})$ toward the previous body $\left(E_{0}\right)$. The quantum of this radiation will carry a linear momentum of magnitude $p=h v / c$, where $h$ : Planck's constant, $v$ : wave's frequency. Now, if there is a force affecting this quantum along it its path in (r), it will mean that the momentum will change with time as long as the quantum

passing through ( $\mathbf{r}$ ), or $\vec{F}=d \vec{p} / d t>0$ which represents the physical effect of the force. Therefore there will be a shift in the frequency associated with the shift in the momentum as $\Delta p=h \Delta v / c$. This shift in the frequency can be interpreted as a physical effect of gravitational time dilatation.

\section{THE EXPERIMENTAL ASPECTS OF SAT}

Initially, any evidence that could be used to verify the existence of ether can be classified into two different categories:

Direct evidence, which detects the existence of the ether directly.

$>$ Indirect evidence, which fails to detect the ether directly, but instead of this, it detects the physical consequences for the existence of the medium.

Till now, there is no any direct evidence that could be used to verify the existence of the ether. This section demonstrates two indirect evidences for the existence of the multi-rest frames medium.

\subsection{SAT and the Behavior of Light}

At the beginning of the $19^{\text {th }}$ century, A British physicist named Thomas Young provided a clear demonstration of the wave nature of light. Young showed that, under appropriate conditions, light rays can interfere with each other, which is a characteristic feature of waves. [12].

The definition of a wave is that it represents a disturbance which propagates from one place to another. The disturbance itself occurs in a preexisting physical entity, which means that waves cannot propagates in empty spacetime except in one situation, if they are a disturbance in the space-time itself (Gravitational waves), and in this particular situation, the space-time itself will represent the medium. Therefore, this definition of the wave is not restricted to the mechanical waves, but also includes field waves. Albert Michelson and Edward Morley carried out at the Case School of Applied Science in Cleveland, Ohio, a careful and accurate experiment. They compared the 
speed of light in two beams at right angles to each other. As the Earth rotates on its axis and orbits the Sun, the apparatus moves through the ether with varying speed and direction. The Michelson-Morley experiment, as it became known, showed that the speed of light is the same in all directions. It was as if light always traveled at the same speed relative to where one was, no matter how fast and in which direction one was moving.

The privilege of SAT is that it accounts well for the experimental findings of Thomas Young's double slit experiment, since the ether itself represents the medium for light propagation and its requirement results from the definition of a wave in physics. Therefore it takes the wave properties of light into account.

SAT also accounts for the experimental findings of Michelson-Morley experiment, since the ether is regarded as multi-rest frames medium, and therefore the light will propagates at the same speed relative to where one was, no matter how fast and in which direction one was moving. Therefore the findings of both Thomas Young and Michelson-Morley experiments represent indirect evidence for the existence of multi-rest frames medium in the universe.

The only serious competitive theory for SAT in explaining the findings of the previous experimental results is the theory that was introduced in 1905 by the physicist Albert Einstein. The theory which is today known as the special theory of relativity (STR). STR accounts well for the findings of Michelson-Morley experiment, since it regards the constancy of the speed of light for all inertial observers as a postulate or a principle in the theory, but the main problem with STR is that it fails to account for wave properties of light ( Thomas Young's findings), since it ignores the wave's definition as a disturbance that propagates in a preexisting physical entity (medium), and this in turn results in the occurrence of the interference pattern of light.

In the early $20^{\text {th }}$ century, in order to accept STR, many physicists were enforced to come up with a contradictory assumption about light which is a wave that travels in empty space-time. The contradiction with this assumption is that it represents a clear violation to the wave definition in physics. Besides that, SAT gives the same mathematics of the STR, which means that all the testable predictions, and their verifying experiments regarding the STR are still hold in SAT. This is not surprising, since the two postulates of STR are a superficial reflections of the SAT first postulate which is the existence of equivalent multiple rest frames in ether.

\subsection{SAT and the Space-time Intervals}

\subsubsection{Defining the Ether Experimentally}

Now let us consider the second indirect evidence of ether. Initially, let us assume that the ether is really existed in nature, but not the ether which is described by SAT, but instead is the old traditional ether model, which represents a uni-rest frame medium (an ether with only one rest frame).

By considering two observers, observer (1) is in rest frame with respect to ether, therefore he measures the value (c) for the speed of light in all directions (Figure18). Observer (2) is moving with speed (v) with respect to observer (1) and ether, therefore he measures the value $c^{\prime}=c-v$ for a propagating light signal in the same direction of his movement.

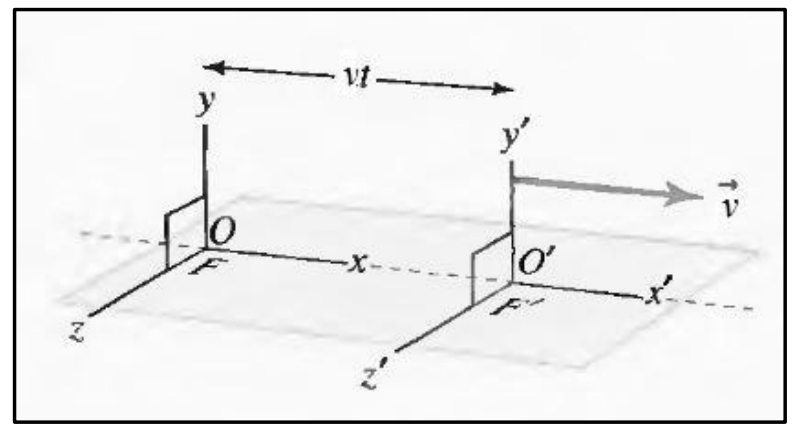

Fig18

Now, let us consider the ether as multi-rest frames medium as described by SAT. By a simple comparison, the major difference between these two different models of ether is that a multi-rest frames medium has a specific physical action which is conserving a constant value for the speed of light propagation for the two observers in the example above, which means that both observers will measure the same speed of light as $c^{\prime}=c$. This equation means that 
both space $(\Delta x)$ and time ( $\Delta t)$ intervals should be curved or changed according to values which are determined by Lorentz transformation:

$$
\begin{aligned}
& \Delta x^{\prime}=\frac{\Delta x-v t}{\sqrt{1-v^{2} / c^{2}}} \\
& \Delta t^{\prime}=\frac{\Delta t-\frac{v \Delta x}{c^{2}}}{\sqrt{1-v^{2} / c^{2}}}
\end{aligned}
$$

Therefore, the ether's action which is conserving a constant speed value for light propagation for both observers, simply means curving or changing both space and time interval with the magnitude that keeps the speed of light constant for all observers. As a result of this, the ether will represent from an experimental point of view:

A medium or a physical entity that curves both space and time intervals with motion according to the value that is required by Lorentz transformation.

$>$ A medium or a physical entity that should be existed everywhere, since it carries light.

Now, let us consider another example. A spacecraft traveling with a speed $v$ from one star to another. There are two observers: one on the Earth and the other in the spacecraft. The observer at rest on the Earth (and also assumed to be at rest with respect to the two stars) measures the distance between the stars to be the proper length $(\Delta x)$. According to this observer, the time interval required for the spacecraft to complete the voyage is $\left(\Delta t^{\prime}=\Delta x / v\right)$. The passages of the two stars by the spacecraft occur at the same position for the space traveler. Thus, the space traveler measures the proper time interval $\Delta t$. Because of time dilation, the proper time interval is related to the Earth-measured time interval by $\left(\Delta t=\Delta t^{\prime} / \gamma\right)$. Where $(\gamma)$ is Lorentz's factor. Because the space traveler reaches the second star in the time $(\Delta t)$, he or she concludes that the distance $\left(\Delta x^{\prime}\right)$ between the stars is:

$$
\Delta x^{\prime}=v \Delta t=v \frac{\Delta t^{\prime}}{\gamma}=\frac{\Delta x}{\gamma} .
$$

The conclusion from the example of the spacecraft is that space and time are intimately connected in nature, Therefore, curving of time intervals with motion cannot occur without curving space intervals. This will mean that any physical entity that curves time with motion, it will also be a physical entity that curves space with motion. From the earlier discussion, the ether will be the physical entity that performs this physical action.

\subsubsection{A new Definition to the Inertial Mass}

Experimental detection of a structure that is capable of curing space-time intervals with motion is possible, but it requires the following information to be correct, which is all the elementary particles are massless particles or lack the rest mass. The inertial rest mass $\left(m_{0}\right)$ for any particle is defined simply as the resistance of the particle to motion which appears when applying a force on it from a rest state. Mathematically, it represents the ratio between magnitudes of the applied force (F) and the resultant acceleration (a) $m_{0} \equiv F / a$.

From the above equation, when the value of $\left(m_{0}\right)$ goes very small, the value of resistance which experienced during particle's acceleration goes very small too. Therefore, when $\left(m_{0}=0\right)$, this means that this particle lacks any resistance to motion when accelerating it. But in this situation the laws of physics require that this particle to be moving with a constant speed which is equivalent to the speed of light in the presumed "vacuum".

The previous information will represent a contradiction with the practical observations, since elementary particles do have a detectable value of a rest mass, and therefore, they move with a speed less than that of light. This contradiction between the previous information and the practical observation, is simply solved by assuming that the resistance to motion which appears when applying a force on an elementary particle does not come from the particle itself(since it is massless, and therefore lacks any resistance), but comes from external source which affects the particle. Now, let us call this source (S), and from here the definition of the inertial rest mass should be changed to the resistance of (S) to the particle's motion, which appears when applying a force on the particle from a rest state.

The value of resistance which is provided by $(\mathrm{S})$ does not represent a constant quantity for a specific particle, since the inertial mass ${ }^{1}\left(m_{i}\right)$ changes with the speed of the particle according to [13]:

$$
\begin{gathered}
m_{i} \equiv \frac{F}{a}=\left(\frac{d p}{d t}\right)\left(\frac{d t}{d v}\right)=\frac{d p}{d v}= \\
m_{i}=\frac{m_{0}}{\left(1-v^{2} / c^{2}\right)^{\frac{3}{2}}}
\end{gathered}
$$




\section{American Research Journal of Physics, Volume 1, Issue 1, 2015}

ISSN 2380-5714

This occurs because the value of the particle's linear momentum (p) changes with speed according to:

$$
\vec{p}=m_{r}(v) \vec{v}=\frac{m_{0} \vec{v}}{\sqrt{1-v^{2} / c^{2}}}
$$

Where $\left(m_{r}\right)$ is called the relativistic mass of the particle. From here $(\mathrm{S})$ will represent a physical entity that:

$>$ Curves or changes the particle's inertial mass with motion from the value $\left(m_{0}\right)$ to the value $\left(m_{i}\right)$.

$>$ Existed everywhere in space, since the inertial mass of the elementary particles are experienced anywhere in space-time.

It is important to know that the relativistic mass $\left(m_{r}\right)$ which appears in (25). Is different from the inertial mass $\left(m_{i}\right)$. The two masses are measured with the same units (kilogram), but their definitions are different. For a linear movement, the relativistic mass $\left(m_{r}\right)$ is defined as the ratio between the magnitudes of the linear momentum and the velocity of the particle $m_{r} \equiv p / v$. It is also defined as the ratio between the particle's energy $(E)$ and the square of the speed of light $m_{r} \equiv E / c^{2}$. Therefore, massless particles lack the inertial mass, but they do have a relativistic mass, since they have a linear momentum and energy.

The two masses are equivalent when the particle's speed is zero as $m_{r}=m_{i}=m_{0}$. But when the particle is moving the relation between the two is given by:

$$
m_{r}=\frac{p}{v}=\frac{\int_{0}^{p} d p}{v}
$$

From (25), the magnitude of $(d \mathbf{p})$ is given by $d p=m_{i}(v) d v$. Now, by substituting this in (2) this gives:

$$
m_{r}=\frac{\int_{0}^{v} m_{i}(v) d v}{v}
$$

Or, another relation is obtained, when substituting the magnitude of (25) directly into the definition of the inertial mass as:

$$
m_{i}=\frac{d p}{d v}=\frac{d\left(m_{r}(v) v\right)}{d v}
$$

Therefore, when (S) changes the value of the inertial mass from $\left(m_{0}\right)$ to $\left(m_{i}\right)$, it also changes the value of the relativistic mass from $\left(m_{0}\right)$ to $\left(m_{r}\right)$.

\subsubsection{A Simple Experiment for Consideration}

Now, let us consider the following experiment. Consider a clock composed of a particle, (Figure 19) moving along vertical axis $(\mathbf{y})$, with a constant velocity, which travels a specific displacement $(\Delta \mathbf{y})$ in a period of time $(T)$. For an observer which he at rest with respect to this clock, the time interval is $T=\Delta y / v_{y}$. The linear momentum of the particle in $(\mathbf{y})$, is given by:

$$
\vec{p}_{y}=\frac{m_{0} \vec{v}_{y}}{\sqrt{1-v_{y}^{2} / c^{2}}}=m \vec{v}_{y}
$$

This will mean that the time interval will be $T=\Delta y / v_{y}=\Delta y m / p_{y}$. Now, by applying an impulse with a force (F) on the particle along direction of the horizontal axis $(\mathbf{x})$. The particle will acquire a velocity along the $(\mathbf{x})$ axis.

Since the clock now is moving along the $(x)$ axis with speed $\left(v_{x}\right)$, the time interval that now will be required for the particle to pass the same $(\Delta \mathbf{y})$ interval since $\left(y=y^{\prime}\right)$, will be $T^{\prime}=\Delta y / v_{y}^{\prime}$. Also, since the impulse was in the $(\mathbf{x})$ direction, and not $(\mathbf{y})$. This means that the magnitude of the momentum $\left(\mathbf{p}_{y}\right)$ will be conserved. This gives :

$$
T^{\prime}=\frac{\Delta y}{v_{y}^{\prime}}=\frac{\Delta y m^{\prime}}{p_{y}}
$$

The relation between $(T)$, and $\left(T^{\prime}\right)$ is given by the time dilatation formula $T^{\prime}=T / \sqrt{1-v_{x}^{2} / c^{2}}$. Now, by recalling that the ether represents the structure that curves space-time intervals with motion. Therefore, in this particular experiment, if the ether is really exist, it will represent a structure that curves or changes the time interval 
from $\left(\frac{\Delta y m}{p_{y}}\right)$, to $\left(\frac{\Delta y m^{\prime}}{p_{y}}\right)$, which simply means changing the value of the relativistic mass from $(m)$ to $\left(m^{\prime}\right)$, since the values of $(\Delta y)$, and $\left(p_{y}\right)$, will remain constant with motion. The value of $\left(m^{\prime}\right)$ is given by:

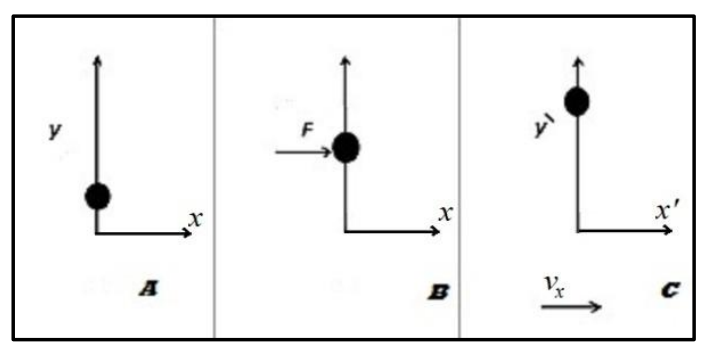

Fig19. (A) the particle is moving along the (y) axis. (B) An impulse is applied to the particle along the horizontal axis $(\boldsymbol{x}) .(C)$ The particle now is moving in the $x$ direction with velocity $\left(\boldsymbol{v}_{x}\right)$

$$
m^{\prime}=\frac{m}{\sqrt{1-v_{x}^{2} / c^{2}}}=\frac{m_{0}}{\sqrt{1-v_{y}^{2} / c^{2}} \sqrt{1-v_{x}^{2} / c^{2}}}=\frac{m_{0}}{\sqrt{1-v^{2} / c^{2}}}
$$

where: $v^{2}=v_{x}^{2}+v_{y}^{\prime 2}=v_{x}^{2}+v_{y}^{2}\left(1-v_{x}^{2} / c^{2}\right)$

From the relations which are given in (27) and (28), when the ether changes the value of the relativistic mass from $(m)$ to $\left(m^{\prime}\right)$, it also changes the value of the inertial mass from $(m)$ to $\left(m_{i}\right)$. Where $\left(m_{i}\right)$ is equals to:

$$
m_{i}=\frac{d p_{x}}{d v_{x}}=\frac{m}{\left(1-v_{x}^{2} / c^{2}\right)^{\frac{3}{2}}}
$$

This physical action of ether represents the same physical action of the unknown physical entity (S) which was introduced earlier. This in turn will lead us to conclude that in this particular experiment, they represent the same physical entity. From here, the definition of the inertial mass will be changed to the resistance of the ether to a particle's motion, which appears when applying a force on the particle. Also since space and time are intimately connected as discussed in (4.2.1), this will result in that $(\mathrm{S})$ will be a structure that curves both space and time intervals with motion.

\subsubsection{Why it is Indirect Evidence?}

Now, let us consider the final point in this paper, which is the relation between the amplitude of a wave, and its carrying medium. Let us take the sound waves which propagate in air as an example. The amplitude of the sound wave is pressure difference. The relationship between the amplitude (pressure), and the medium (air) is that the amplitude represents a physical value in the medium which is the differences in air pressure measured by a specific physical units ( Newton/ square meter).

By considering another example, a wave which propagates in a string. Here the amplitude is a displacement $(\Delta \mathbf{y})$. The relation between the amplitude (displacement) and the medium (string) is that the amplitude represents a physical value in the string which is the difference in string position along (y), which is measured with specific physical units (meter). Generally, the relation is the same between any other wave's amplitude and its carrying medium.

Therefore, we have to conclude that the amplitude of any wave represents a specific physical value in its carrying medium. When this concept is applied for ether, the value of the amplitude of the electromagnetic (EM) waves (E, B) will represent physical values in ether which are measured with the physical units (Newton/Coulomb, and Tesla respectively). The difference here between the ether and the other mechanical mediums, is that the ether has a multiple values of the amplitudes as explained in section (3.2). Therefore, each amplitude represents a different field measured with a different physical units, which in turn represents a different physical value in ether.

Now, the previous experiment introduces another physical value in the ether which is the inertial mass, or the ether's resistance to particle's motion which appears when applying a force on that particle. This ether's resistance is measured with a specific physical unit (kilogram). Now, by recalling the previous conclusion (the amplitude of any wave represents a specific physical value in its carrying medium), from here, we have to introduce another 
amplitude in ether, the $(H)$ amplitude. This represents a specific physical value in ether which is the ether's resistance to a particle's motion which appears when applying a force on that particle measured with kilograms.

Therefore, a particle with huge inertial mass, will be associated with a huge value of the amplitude $(H)$ or field $(H)$. In contrast with a massless particle, this will be associated with the minimum value of the amplitude or field $(H)$. Now, by recalling the previous experiment, the unknown physical structure $(\mathrm{S})$, will be equivalent to $(H)$ field, since it represents the value of the inertial mass in ether, but not the ether directly. Therefore, the definition of the inertial mass will be changed to a more specific form which is the resistance of the $(H)$ field to a particle's motion which appears when applying a force on the particle, since the value of the inertial mass is governed by the value of the amplitude $(H)$.

This means that after all, the ether will not be detected by the previous experiment, but instead something else will be detected which is the $(H)$ field, but the validity of the previous experiment is that it detects the existence of a structure that is capable of curving (changing) the values of space and time intervals with motion, which is a property of ether as explained earlier.

Therefore we are enforced to say that the ether or a multi-rest frames medium does exist in order to account this physical action, because if we neglect the ether and consider only the existence of the $(\mathrm{H})$ field, the previous physical action cannot be accounted, since curving(changing) space-time intervals with motion is not a physical property for the $(\mathrm{H})$ field, but is a physical property for ether.

\section{DISCUSSION AND CONCLUSIONS}

We have shown that the first postulate of SAT gives physical consequences that can accounts for the results of Michelson-Morley experiment. Consequences like length contraction, and time dilatation with motion, which demonstrates that SAT is consistent with Lorentz's transformation. By making a comparison between SAT and Lorentz's ether theory (LET) regarding the notion of ether itself, we will find that the constancy of the speed of light with motion in SAT is considered to be a consequence of a physical character of ether which is the existence of multiple rest frames. This is in contrast with LET which fails to provide a reliable physical meaning or interpretation for the constancy of the speed of the light with motion.

Also by making another comparison between SAT and the special theory of relativity (STR)[14]. From the prospect of the first SAT postulate, we will find that both SAT and STR share the same mathematics, therefore, both give the same physical results, but the privilege of SAT is that it can account for the wave properties of light. This is because the ether represents the necessary medium for light propagation which is consistent with the wave definition in physics as propagating disturbance in which the medium represents the physical entity where the disturbance occurs. On the other hand STR fails to account the problem of light propagation since it ignores the light medium (ether).

Einstein himself knew that his theory (STR) has this problem, but he choose to ignore it, and many physicists of the $20^{\text {th }}$ century simply followed his steps. In 1938 a book which was written by him, and A. Infeld concerning the problem of light propagation through space, They mentioned that [15]:

"Our only way out seems to be to take for granted the fact that space has the physical property of transmitting electromagnetic waves, and not to bother too much about the meaning of this statement".

Clearly, SAT solves this problem very simply as discussed earlier. Furthermore, all the testable predictions and the experiments used to confirm STR are still valued for SAT. Also the concept of the relative motion is still hold in SAT and this is not surprising since the two postulates of STR are just a superficial reflections of the SAT first postulate which is the existence of a multiple equivalent rest frames in ether.We have also shown that the contradictory wave-particle duality is eliminated in SAT simply by assuming that the kinetic energy and the potential energy are the only forms of energy for the ether's wave. Therefore, only the wave theory is needed to describe the ether's waves. This assumption also requires that the rest energy of a body to be defined as a kinetic energy, which will result in existence of physical effects that is equivalent to the gravitational effects around the body.

Finally, the existence of ether as multi-rest states medium accounts for the wave properties of light which represents Thomas Young's findings, and for the constancy of the speed of light for all inertial observers which represents the finding of Michelson-Morley experiment. We have also shown a simple experiment that reveals the existence of a structure in the universe that is capable of curving (changing) space-time intervals with motion for an observer. This physical action is accounted by the existence of multi-rest states medium. 


\section{REFERENCES}

[1] Sehutz, B. (2003) "Gravity from the Ground up”. Cambridge university press. , pp. 309-330.

[2] Fokko, J. D.(2004), Lenses and Waves: Christiaan Huygens and the Mathematical Science of Optics in the 17th Century, Kluwer Academic Publishers.

[3] Whittaker, E.T. (1910), 1. Edition: A History of the theories of aether and electricity, Dublin: Longman, Green and Co.

[4] Michelson, A. A. \& Morley, E. W. (1887), On the Relative Motion of the Earth and the Luminiferous Ether, American Journal of Science 34(203): 333-345.

[5] Lorentz, H. A. (1899), Simplified theory of electrical and optical phenomena in moving systems, Proc. Acad. Science Amsterdam I: 427-443

[6] Lorentz, H. A. (1904), Electromagnetic phenomena in a system moving with any velocity less than that of light, Proc. Acad. Science Amsterdam IV: 669-678

[7] Lorentz, H.A (1916), The theory of electrons, Leipzig \& Berlin: B.G. Teubner.

[8] Pierce, J. R. (1974). Almost All About Waves .The Massachusetts institute of Technology, p 144.

[9] Beiser, A.(2003) Concepts of Modern Physics , $6^{\text {th }}$ edition, McGraw Hill companies, pp.67-68.

[10] Fishbane, P.M, Gasiorowicz, S.G, and Thornton, S.T."Physics for Scientists and Engineers with modern physics", Pearson Prentice Hall.

[11] Isam, A. ( 2013). Concerning the Existence of a discrete Space, Jordan Journal of physics, Vol.6, No.2, pp. 73-77..

[12] Shamos, M (1959). Great Experiments in Physics. New York: Holt Rinehart and Winston. pp. 96-101.

[13] Okun, L. B. (1989). The Concept of Mass. Physics Today 42 (6): 31-36.

[14] Einstein, Albert (1961), Relativity: The Special and the General Theory, New York: Three Rivers Press (published 1995).

[15] Einstein, A\& Infeld, L(1938) .Evolution of Physics. Cambridge University Press, London. ,p 159.

How to cite this paper: Author 1, Author 2 and Author 3 (2014) Paper Title. *********,*,**_**. http://dx.doi.org/10.4236/jmp.2014.***** 